\title{
Il campione microbiologico: applicazione pratica di uno strumento per monitorare la qualità della fase preanalitica
}

\author{
Laura Pederzolli \\ Laboratorio di Microbiologia e Immunologia - Ospedale "S. Chiara"Trento
}

La fase preanalitica rappresenta un momento cruciale nell'ambito del processo di laboratorio, durante la quale si compie il $46 \%$ degli errori. Tali errori spesso possono risultare estremamente critici, fino al punto di compromettere gravemente la qualità del servizio recato al Paziente.

La fase preanalitica, per la sua stessa natura, rappresenta anche un fondamentale momento di interfaccia tra il laboratorio e le altre parti interessate al processo (stakeholders).

Occuparsi di fase preanalitica, pertanto, significa occuparsi anche di come il laboratorio è in grado di rispondere ai bisogni di chi vi si rivolge e di come si integra all'interno di un processo più complesso di cui fa parte, cioè il processo aziendale.

Ogni anello della "catena aziendale" deve anche e soprattutto essere funzionale alla catena.

L'Azienda Provinciale per i Servizi Sanitari della Provincia Autonoma di Trento, nella dichiarazione della propria Vision fa esplicito riferimento ai processi aziendali e organizzativi che devono essere coordinati:

"Vogliamo diventare un'organizzazione che modella in modo coordinato i processi aziendali e organizzativi attorno alle legittime esigenze ed aspettative dei cittadini, conciliando la centralità dei cittadini stessi con le esigenze ed aspettative, altrettanto legittime, di tutte le altre parti interessate al funzionamento della nostra azienda"

fonte: Programma di Sviluppo Strategico - APSS

Provincia Autonoma di Trento - gennaio 2001.

Quali strumenti, dunque, ci possono aiutare a gestire la Qualità nella fase preanalitica?

Alcuni strumenti fanno parte ormai da molti anni della buona pratica di laboratorio. Un esempio è la carta di controllo, nella sua più diffusa applicazione: la carta di Leavy-Jennings. È uno strumento ormai consolidato, che ha subito una sua evoluzione e anche numerose critiche da parte di alcuni Autori.

Non si può comunque negare che abbia svolto un ruolo importante e abbia contribuito nel tempo a ridurre l'errore analitico, come supporto del Controllo di Qualità.

Si possono comunque studiare altre interessanti applicazioni anche per la fase preanalitica (esem- pio: monitoraggio dei tempi di attesa di una prestazione che necessita di appuntamento, monitoraggio dei tempi di attesa di fornitura di materiali o reagenti per il laboratorio...).

Il diagramma causa-effetto, non così diffuso in laboratorio, si pone come obiettivo quello di fornire una visione sintetica e completa delle cause del fenomeno, negativo o positivo, che si intende studiare (effetto). Nelle realtà produttive si tende a raggruppare le cause per tipologia; generalmente i gruppi sono: il personale, le strutture, i metodi, le attrezzature.

Il diagramma di flusso rappresenta graficamente la sequenza di eventi che caratterizza un processo. La flow-chart può essere un modo sintetico, semplice e immediato per rappresentare una procedura di laboratorio.

Un altro esempio di strumento per la gestione della Qualità è il diagramma di Gantt: serve per rappresentare la sequenza temporale delle attività di un progetto (esempio: tabellone della turistica, attività di formazione e aggiornamento, trasloco, pulizie e allestimento del nuovo laboratorio...).

L'Analisi di Pareto si pone come obiettivo quello di individuare delle priorità sulle quali agire, senza perdersi su fattori meno significativi e di scarsa rilevanza.

Poche voci, infatti sono in grado di rappresentare la maggior parte del fenomeno.

L'Analisi si Pareto si basa su un'osservazione empirica piuttosto semplice: tra tutti gli errori possibili, solo un gruppo costituito dal $20 \%$ di essi è in grado di determinare $1{ }^{\prime} 80 \%$ dei prodotti viziati (si chiama anche regola dell'80-20). In linea del tutto teorica, agendo quel $20 \%$ di cause di errore si dovrebbe eliminare $1^{\prime} 80 \%$ di errore sul prodotto.

La sua stessa definizione ci ha indotti a presumere che si potes se trattare di uno strumento utile ad abbattere l'errore della fase preanalitica. Abbiamo quindi deciso di sperimentare una sua applicazione nell'ambito della nostra realtà quotidiana. Nella nostra applicazione dell'Analisi di Pareto ci siamo riferiti ai principi del ciclo di Deming o PDCA (Plan, Do, Check, Act). Si tratta di una rappresentazione schematica di come qualsiasi attività routinaria o straordinaria dovrebbe esse- 
re affrontata affinché possa produrre una opportunità di miglioramento. In questo approccio ogni attività, dalla più semplice alla più complessa, dovrebbe essere pianificata, valutandone l'opportunità e dotandosi degli strumenti necessari, dovrebbe essere attuata nel modo pianificato, si dovrebbe controllarne e verificarne $i$ risultati e infine, attuare delle strategie di miglioramento. $\grave{E}$ un modello estremamente dinamico perché ogni attività ed ogni evento deve rientrare nel ciclo in modo tale che tutto sia sempre orientato a migliorare.

Tornando all'Analisi di Pareto, quali Non Conformità (NC) costituiscono il $20 \%$ di errore in grado di spiegare la maggior parte dell'errore preanalitico?

Per rispondere a questa domanda, nella fase di "Plan" o pianificazione abbiamo predisposto due schede di rilevazione delle $\mathrm{NC}$, una per la parte più propriamente amministrativa di segreteria, l'altra per il laboratorio di batteriologia.

Per la segreteria abbiamo individuato le seguenti NC: scheda ottica di accettazione degli esami senza anagrafica del Paziente, scheda con anagrafica incompleta, esami non barrati, esami barrati erroneamente, scambio di etichetta anagrafica, campione senza scheda di accettazione, altra tipologia da specificare.

Per il laboratorio abbiamo distinto le NC relative alla fase di raccolta (contenitore errato, quantità errata, materiale non idoneo, contenitore sporco o non conforme alle norme di sicurezza); NC relative alla fase di conservazione (urine e liquidi non conservati in frigo, tamponi e fiasche non a temperatura ambiente); $\mathrm{NC}$ delle modalità di trasporto (mancanza di terreno di trasporto ove richiesto, tempi di consegna troppo lunghi per errori di smistamento dei campioni), non idoneità dei contenitori rispetto alle norme di sicurezza; campione senza scheda di accettazione, campione non pervenuto, altra tipologia di $\mathrm{NC}$ da specificare.

Le schede di rilevazione delle NC sono state predisposte solo allo scopo di raccogliere i dati da sottoporre ad analisi di Pareto e facendo riferimento alle linee guida che il laboratorio ha prodotto, aggiornato nel gennaio 2001 e distribuito a tutti i propri utenti (MODALITÀ DI PRELIEVO E CONSERVAZIONE DEI CAMPIONI PER RICERCHE BATTERIOLOGICHE); il laboratorio, conformemente a quanto previsto dalla norma ISO 15189 dovrà dotarsi di un sistema di gestione delle NC molto più articolato e complesso.

Nella fase "Do" tutti gli operatori del laboratorio (personale tecnico ed amministrativo) per il periodo compreso tra maggio e agosto 2003 ha registrato sulle schede tutti gli eventi riferibili ad errore della fase preanalitica, ottenendo i seguenti risultati:

\begin{tabular}{|c|c|c|}
\hline & TIPOLOGIA NON CONFORMITÀ & N. casi \\
\hline A & Materiale non idoneo & 89 \\
\hline B & Campione non pervenuto & 82 \\
\hline C & $\begin{array}{l}\text { Camp. sporco o non conforme } \\
\text { alle norme di sicurezza }\end{array}$ & 72 \\
\hline D & Contenitore errato & 45 \\
\hline E & Campione senza scheda di accettazione & 29 \\
\hline$\underline{F}$ & Scheda con anagrafica incompleta & 29 \\
\hline G & Esami non barrati & 24 \\
\hline$\underline{\mathrm{H}}$ & Esami barrati erroneamente & 24 \\
\hline 1 & Bar-code fiasca coperto da etichetta & 18 \\
\hline $\mathrm{L}$ & $\begin{array}{l}\text { Tempi di consegna eccessivi } \\
\text { (errore smistamento) }\end{array}$ & 11 \\
\hline$M$ & Quantità errata & 8 \\
\hline$\underline{N}$ & Campione anonimo & 7 \\
\hline O & Non idoneità dei contenitori (sicurezza) & 5 \\
\hline$\underline{P}$ & Scheda senza anagrafica & 4 \\
\hline $\mathrm{Q}$ & Assenza di terreno di trasporto ove richiesto & 3 \\
\hline$\underline{\mathrm{R}}$ & Scheda di accettazione sporca & 3 \\
\hline$\underline{S}$ & Fiasche non a Temperatura ambiente & 1 \\
\hline $\mathrm{T}$ & Urine e liquidi non in frigo & 0 \\
\hline$\underline{U}$ & Scambio di etichetta anagrafica & 0 \\
\hline
\end{tabular}

II Grafico di Pareto, nella fase di verifica (Check) ha dimostrato che 6 NC sono in grado di determinare più dell' $80 \%$ dell'errore nella fase preanalitica del nostro laboratorio. II grafico è una distribuzione di frequenza con la curva delle percentuali cumulate sovrapposta.

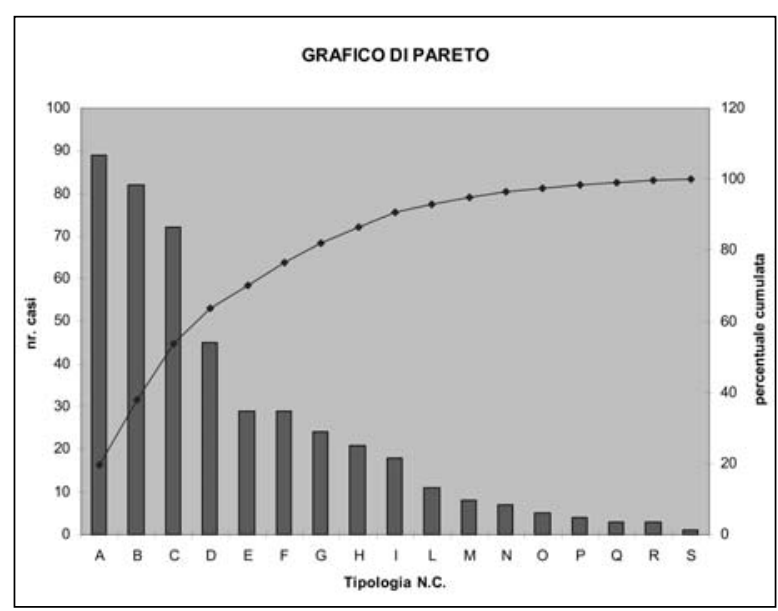

La NC più frequente "materiale non idoneo" è riconducibile agli errori delle modalità di raccolta. Sono tutti i materiali non appropriati per l'esecuzione dell'indagine richiesta e sono per la grande maggioranza escreati non rappresentativi delle basse vie respiratorie, contaminati da materiale salivare: 56 campioni con la richiesta di ricerca microscopica e colturale di Micobatteri e 23 per germi comuni. Ci sono poi 7 vaginali con richieste non appropriate (es, Chlamydia su sperma, micoplasma su essudato vaginale...), 2 campioni per indagine parassitologica, 1 liquido addomina- 
le coagulato cioè non adeguatamente pretrattato con anticoagulante.

La seconda NC più frequente si riferisce ai campioni non pervenuti, ovvero tutti i campioni assenti, per i quali è presente un'accettazione. Si tratta per la maggior parte di prenotazioni di secondi o terzi campioni ( 21 campioni di feci per indagine parassitologica e 16 escreati) che non vengono più inviati al laboratorio. Con l'invio del primo campione si procede cioè alla richiesta contemporanea di un secondo e di un terzo campione che non viene più inviato. Nel periodo di osservazione in studio sono inoltre risultati non pervenuti 13 materiali per micobatteri, 9 essudati vaginali, 8 emocolture, 7 urine, 3 cateteri venosi centrali, 3 materiali vari, 2 tamponi faringei.

La terza NC riguarda le modalità di raccolta. Si tratta dei campioni sporchi e/o non conformi alle norme di sicurezza e si è verificata per 56 emocolture (ciascuna delle quali composta da 6 flaconi, per una quantità di flaconi non conformi superiore a 300), 4 materiali per $\mathrm{BK}, 3$ campioni di feci per coprocoltura o esame parassitologico, 3 materiali vari, 2 urine, 2 tamponi vaginali, 1 tampone faringeo, 1 escreato. Questa NC pur occupando la terza posizione in termini di numerosità rappresenta sicuramente la più grave. Il messaggio più importante che l'Analisi di Pareto ci sottopone con estrema evidenza è che molti operatori ignorano le più semplici norme di sicurezza ed il rischio che un campione sporco rappresenta per tutti gli addetti dei reparti, del trasporto e del laboratorio, nonostante le normative vigenti siano molto chiare in termini di responsabilità. A questo punto si impone un'adeguata azione preventiva.

In 45 casi si è verificato l'errore nella scelta di un contenitore diverso da quello raccomandato dal laboratorio.

Le altre NC riguardano errori nella gestione della scheda ottica per l'accettazione dei campioni.

Quali sono le ricadute concrete che, a questo punto l'analisi di Pareto deve indurre? Ecco la fase finale del ciclo PDCA e cioè l'act (mettere in pratica, far funzionare, agire). L'analisi di Pareto ha dimostrato la necessità di sollecitare le parti interessate ad una maggiore attenzione circa le modalità di raccolta degli escreati, la corrispondenza tra test richiesti e campioni inviati, ma soprattutto le modalità di raccolta del campione in condizioni di sicurezza. Le azioni preventive che si devono intraprendere possono essere molteplici ma le norme ISO 15189 raccomandano il confronto diretto con il personale coinvolto attraverso degli audit.

Dall'esperienza condotta ci sembra di poter individuare i seguenti svantaggi dell'analisi di Pareto:

- Non considerare la criticità delle NC ma solo la numerosità

- Esigere lunghi periodi di osservazione e minuziosa registrazione delle NC.

- Prevedere l'applicazione di Azioni Preventive mirate ed efficaci (rivolte alle priorità), che producono miglioramento a lungo termine (da verificare con ulteriore analisi di Pareto?)

Per tutte queste ragioni riteniamo che l'Azione Correttiva immediata di fronte ad una singola e specifica $\mathrm{NC}$, indipendentemente dalla criticità della stessa, sia irrinunciabile e rappresenti un momento privilegiato, non certamente per "ricercare e punire i colpevoli" ma per creare opportunità di miglioramento, utili a tutta l'organizzazione. Il ciclo PDCA, infatti, può efficacemente concludersi anche in pochi minuti.

L'azione combinata di correzione e prevenzione delle NC rappresenta una delle strade da percorrere per gestire, controllare, migliorare la Qualità; l'analisi di Pareto può essere uno strumento utile per selezionare le azioni preventive da intraprendere in modo prioritario. La sua sistematica applicazione alla realtà preanalitica del laboratorio dovrebbe efficacemente contribuire ad una gestione orientata al miglioramento continuo. 